\title{
Atypical Presentation of Crimean-Congo Hemorrhagic Fever as Ascending Paralysis with Rhabdomyolysis
}

\author{
Hari Prasad $R^{1}$, Ankur Sharma ${ }^{2}$, Nikhil Kothari ${ }^{3}$, Varuna Vyas ${ }^{4}$, Shilpa Goyal ${ }^{5}$
}

\begin{abstract}
Crimean-Congo hemorrhagic fever (CCHF) is a tick-borne disease caused by a arbovirus. It is asymptomatic in infected animals but a serious threat to the health of individuals. In human, it starts with nonspecific febrile diseases and progresses into severe hemorrhagic syndrome with high-casual fatality. Here, we report a case of CCHF with atypical presentation of ascending paralysis and rhabdomyolysis.

Keywords: Crimean-Congo hemorrhagic fever, Paralysis, Rhabdomyolysis.

Indian Journal of Critical Care Medicine (2020): 10.5005/jp-journals-10071-23347
\end{abstract}

\section{BACKGROUND}

Crimean-Congo hemorrhagic fever (CCHF) is a zoonotic viral illness that is asymptomatic in affected livestock but is a severe risk to human health. It is caused by a virus of family Bunyaviridae and affects mostly adults. The sources of exposure include being bitten by a tick, contacting animal blood or tissues, and drinking unpasteurized milk. ${ }^{1}$ Although ticks often transmit the causative virus, there is also transmission of animal-to-human and human-tohuman. Transmission from person to person may happen, especially when the skin or mucosa membranes are exposed to hemorrhages and tissue. This disease poses a specific danger to farmers and other farm employees, veterinarians, laboratory employees, and hospital staff. Infection happens more frequently in individuals with indoor occupations such as peasants, dairymaids, or forest employees. ${ }^{1,2}$

Patients with CCHF can present with abrupt onset of fever and other nonspecific symptoms, i.e., chills, severe headache, dizziness, photophobia, neck pain, myalgia, and arthralgia. Gastrointestinal symptoms include nausea, vomiting, nonbloody diarrhea, and abdominal pain. ${ }^{1}$ It is followed, after several days, by the hemorrhagic phase. The hemorrhagic stage suddenly develops. Usually it is shorter and lasts 2-3 days on average. Usually it manifests as a petechial rash. Petechias, ecchymoses, and big contusions on the skin and mucous membranes usually follow the rash. Hematemesis, melena, epistaxis, hematuria, hemoptysis, and bleeding from venipuncture sites can also occur. ${ }^{1}$ Recovery usually starts in 10-20 days after onset of the disease in surviving patients. The median fatality rate for cases is $30-50 \%$; however, it has been recorded between $10 \%$ and $80 \%$ in multiple outbreaks. ${ }^{2}$ Some patients die from hemorrhages, hemorrhagic pneumonia, or cardiovascular disturbances.

\section{Case Description}

An 18-year-old male patient came to emergency with symptoms of severe generalized body ache, more pronounced in the lower back with weakness of both legs and inability to walk since 2 days. He had abdominal pain and jaundice since 7 days and was admitted in a private hospital and managed as a hepatitis patient. He was diagnosed of rheumatic heart disease 3 months back with severe mitral regurgitation and severe aortic regurgitation for which valve repair was advised. He does not had any history of fever, vomiting,
1,3,5 Department of Anaesthesiology and Critical Care, All India Institute of Medical Sciences, Jodhpur, Rajasthan, India

${ }^{2}$ Department of Trauma and Emergency (Anaesthesiology), All India Institute of Medical Sciences, Jodhpur, Rajasthan, India

${ }^{4}$ Department of Pediatrics, All India Institute of Medical Sciences, Jodhpur, Rajasthan, India

Corresponding Author: Ankur Sharma, Department of Trauma and Emergency (Anaesthesiology), All India Institute of Medical Sciences, Jodhpur, Rajasthan, India, Phone: +91 9654045653, e-mail: ankuranaesthesia@gmail.com

How to cite this article: Prasad HR, Sharma A, Kothari N, Vyas V, Goyal S. Atypical Presentation of Crimean-Congo Hemorrhagic Fever as Ascending Paralysis with Rhabdomyolysis. Indian J Crit Care Med 2020;24(2):143-144.

Source of support: Nil

Conflict of interest: None

diarrhea, hemoptysis, hematemesis, epistaxis, trauma, and previous surgery at the time of admission.

On examination, his Glasgow Coma Scale (GCS) was 15/15 and was oriented to time, place, and person. He had stable vitals but had bilateral pedal edema. His bilateral lower limb power was $2 / 5$ with hypotonia and hyporeflexia. Upper limb neurological and cranial nerve examinations were normal. He had pansystolic murmur and normal vesicular breath sound on chest auscultation. He was admitted in the intensive care unit for further management where he was evaluated for ascending paralysis and myalgia in suspicion for Guillain-Barré syndrome.

\section{INVESTIGATIONS}

There was leukocytosis of $13,000 / \mathrm{mm}^{3}$ with mild anemia and normal platelet count. The cerebrospinal fluid (CSF) analysis was done, which revealed the normal biochemical report with no albuminocytological dissociation. The nerve conduction study was done, which was also normal. $N$-Acetyl-cystein-(NAC)-activated creatine kinase (CK-NAC) was $3,843 \mathrm{U} / \mathrm{L}$, which was suggestive of rhabdomyolysis. His liver function test showed alanine aminotransferase (ALT) of $2139 \mathrm{U} / \mathrm{L}$, aspartate transaminase (AST) of $2356 \mathrm{U} / \mathrm{L}$, total bilirubin of $2.32 \mathrm{mg} / \mathrm{dL}$, prothrombin time -56.6 seconds, and international normalized 
ratio (INR) - 4.73, which revealed acute fulminant hepatic failure. His serum ammonia level was $431 \mu / \mathrm{dL}$, which was also in favor of hepatic encephalopathy. On the next day, the patient started developing metabolic acidosis with acute kidney injury with rapidly raising urea and creatinine level. He had multiple episodes of hemoptysis, bleeding gums, and petechial rash on the skin. His complete blood counts $(\mathrm{CBC})$ trend showed worsening anemia and thrombocytopenia with increasing total leukocyte count. On suspicion of viral hemorrhagic fever, blood samples for Epstein-Barr virus (EBV), cytomegalovirus (CMV), Parvo B19, and West Nile virus were sent for diagnosis to the National Institute of Virology and were found negative. Malaria, dengue NS1, typhidot, blood culture, scrub antibody, and leptospira antibody also showed negative result. His father was a farmer and there were seven cows out of which one cow died recently, which lead to suspicion of tick-borne hemorrhagic fever. His blood sample was sent to National Institute of Virology for anti-CCHF IgM, which turned out to be positive.

\section{Outcome and Follow-up}

The patient started developing respiratory discomfort and hypoxic episodes. He was intubated and kept on the ventilator support. Hepatic encephalopathy was managed conservatively, acute kidney injury (AKI) was managed by the continuous renal replacement therapy (CRRT), and bleeding was controlled by blood products and tranexamic acid. On day 5 of admission, he had episode of bradycardia and asystole, for which CPR was done and return of spontaneous circulation (ROSC) achieved. Consecutively, he had seven episodes of cardiac arrest in next 3 days and ROSC achieved six times. He died of multiorgan dysfunction and refractory shock.

\section{Discussion}

This disease is endemic in many countries in Africa, Europe, Middle East, and Central Asia with sporadic outbreaks recorded in Kosovo, Albania, Iran, and Turkey. ${ }^{1}$ In India, the first laboratory confirmed case was reported on January 19, 2011, in Gujarat. ${ }^{3}$

Usual presentation of CCHF is fever with nausea, vomiting, abdominal pain, and myalgia in the prehemorrhagic phase followed by bleeding from various sites. But our patient presented as ascending paralysis with no history of fever resembled as Guillain-Barré syndrome and managed accordingly. The diagnosis of CCHF was made after 6th day of illness. Till that, all healthcare providers were unaware that the patient was suffering from CCHF and got exposed to the virus. Then all universal precautions were taken and the dead body was disposed according the World Health
Organization (WHO) recommendations. To avoid infections in hospital settings, stringent infection-control practices, proper floor and air handling in intensive care units, patient isolation, and correct handling of the corpse are essential. ${ }^{3}$ So this case report emphasizes importance of the high index of suspicion of CCHF when the patient presents with atypical symptoms. It could be misdiagnosed and lead to a dangerous massive epidemic. ${ }^{4}$

\section{Learning Points}

- The high-risk personnel in endemic regions should be educated and informed about the threat of CCHF.

- They should go to health centers immediately following any tick bite.

- The patient can present with atypical symptoms.

- If misdiagnosed, it can lead to a dangerous massive epidemic.

\section{Author Contributions}

All authors contributed to the article. Hari P Ramalingam wrote the majority of the article including the background and the case presentation. He was also involved in the treatment and follow-up of the patient. Ankur Sharma wrote majority of the discussion section and the learning points section. He also carried out the literature search and edited the whole article. Varuna Vyas reviewed the article and suggested changes and improvements. She wrote sections of the discussion. Nikhil Kothari was involved in writing the summary, the case presentation, the differential diagnosis, the outcome, and follow-up. He also took part in the patient's management and was the consultant in charge of the critical care.

\section{References}

1. Shayan S, Bokaean M, Shahrivar MR, Chinikar S. Crimean-congo hemorrhagic fever. Lab Med 2015;46(3):180-189. DOI: 10.1309/ LMN1P2FRZ7BKZSCO.

2. Verma R, Khanna P, Prinja S, Rajput M. Crimean-congo haemorrhagic fever: an outbreak in India. Australas Med J 2011;4(11):589-591. DOI: 10.4066/AMJ.2011.701.

3. Mishra AC, Mehta M, Mourya DT, Gandhi S. Crimean-congo haemorrhagic fever in India. Lancet 2011;378(9788):372. DOI: 10.1016/ S0140-6736(11)60680-6.

4. Lahariya C, Goel MK, Kumar A, Puri M, Sodhi A. Emergence of viral hemorrhagic fevers: is recent outbreak of Crimean Congo hemorrhagic fever in India an indication? J Postgrad Med 2012;58(1):39-46. DOI: 10.4103/0022-3859.93251. 